\title{
Algorithms for Solving the Structural Gravity Problem in a Multilayer Medium
}

\author{
E. N. Akimova ${ }^{a, c}$, Corresponding Member of the RAS P. S. Martyshko ${ }^{b, c}$, and V. E. Misilov ${ }^{a}$ \\ Received June 4, 2013
}

DOI: $10.1134 / \mathrm{S} 1028334 \mathrm{X} 13120180$

Of great importance in studying the structure of the Earth's crust is solving the structural inverse gravity problem, which consists in finding the interface between layers with different densities using known density jumps and a gravitational field. This is an illposed problem described by the nonlinear integral first-order Fredholm equation.

In [1], the method of local corrections is applied to determine the structural boundaries. The observed data are processed with the technique proposed by Martyshko and Prutkin [2]: fields for each layer between given depths are calculated and then a modified method of local corrections is applied to find the structural boundary from the corresponding distinguished field.

Non-linear analogs of the methods of the steepest descent, minimum error, and Levenberg-Marquardt method [3], iteratively regularized Newton method [4], and gradient iterative methods with constant damping factors [5] were also used to solve the nonlinear gravity problem in the two-layer and three-layer medium for preliminarily calculated fields.

Here we try another approach based on application of linearized gradient methods with new weight factors. Such an approach makes it possible to find several structural boundaries from the integral equation simultaneously for the sum of fields distinguished from the observed field, thus improving the solution accuracy and decreasing the iteration number and execution time.

Efficient parallel algorithms based on the gradient methods with weight factors were developed and implemented on a multicore Intel processor and NVIDIA Tesla graphic processor unit incorporated in the Uran supercomputer. The structural gravity problem was solved in a four-layer medium for the eastern part of the Middle Urals from real observations.

\footnotetext{
${ }^{a}$ Krasovskii Institute of Mathematics and Mechanics, Ural Branch, Russian Academy of Sciences, Yekaterinburg, Russia

${ }^{b}$ Bulashevich Institute of Geophysics, Ural Branch, Russian Academy of Sciences, Yekaterinburg, Russia

${ }^{c}$ Yeltsin Ural Federal University, Yekaterinburg, Russia
}

Assume that the lower half-space consists of several layers of constant density divided by the sought surfaces $S_{l}(l=1,2, \ldots, L)$, where $L$ is the number of interfaces (Fig. 1). The gravitational effect generated by such a half-space equals the sum of gravitational effects of contact surfaces. Let interfaces be defined by equations $z_{l}=z_{l}(x, y)$, the density jumps on them are $\Delta \sigma_{l}$, and surfaces have horizontal asymptotic planes $z_{l}=H_{l}$, i.e., $\lim _{|x|,|y| \rightarrow \infty}\left|z_{l}(x, y)-H_{l}\right|=0$.

The field of boundary superposition with accuracy to the constant term equals [1]

$$
\begin{gathered}
A(z) \equiv f \sum_{l=1}^{L} \Delta \sigma_{l} \int_{-\infty-\infty}^{\infty} \int_{-\infty}^{\infty}\left(\frac{1}{\sqrt{\left(x-x^{\prime}\right)^{2}+\left(y-y^{\prime}\right)^{2}+z_{l}^{2}(x, y)}}\right. \\
\left.-\frac{1}{\sqrt{\left(x-x^{\prime}\right)^{2}+\left(y-y^{\prime}\right)^{2}+H_{l}^{2}}}\right) d x d y=\Delta g\left(x^{\prime}, y^{\prime}, 0\right)
\end{gathered}
$$

where $f$ is the gravitational constant, $L$ is the number of interfaces, $\Delta g(x, y)=\sum_{l=1}^{L} \Delta g_{l}$.

Discretization of Eq. (1) on the $n=M \times N$ grid with given $\Delta g(x, y)$ and approximation of integral operator $A(z)$ using the quadrature formula yield the right-hand side vector $F(x, y)$ of $M \times N$ dimension, the resultant solution method $z(x, y)=\left[z_{1}(x, y), z_{2}(x, y), \ldots, z_{L}(x, y)\right]$ of $L \times M \times N$ dimension, the matrix of the derivative of operator $A^{\prime}\left(z^{k}\right)^{T}$ of $L \times M^{2} \times N^{2}$ dimension, and the system of nonlinear equations

$$
\tilde{A}_{n}[z]=\tilde{F}_{n} .
$$

The problem is undetermined, since we attempt to find several unknown functions $z_{l}=z_{l}(x, y)$ from the given function $\Delta g(x, y)$.

In this work, the gravity problem in a multilayer medium is solved using linearized iterative gradient methods with new weight factors $\gamma_{i}$ calculated for each component $z_{i}, i=1,2, \ldots, L \times M \times N$ : 


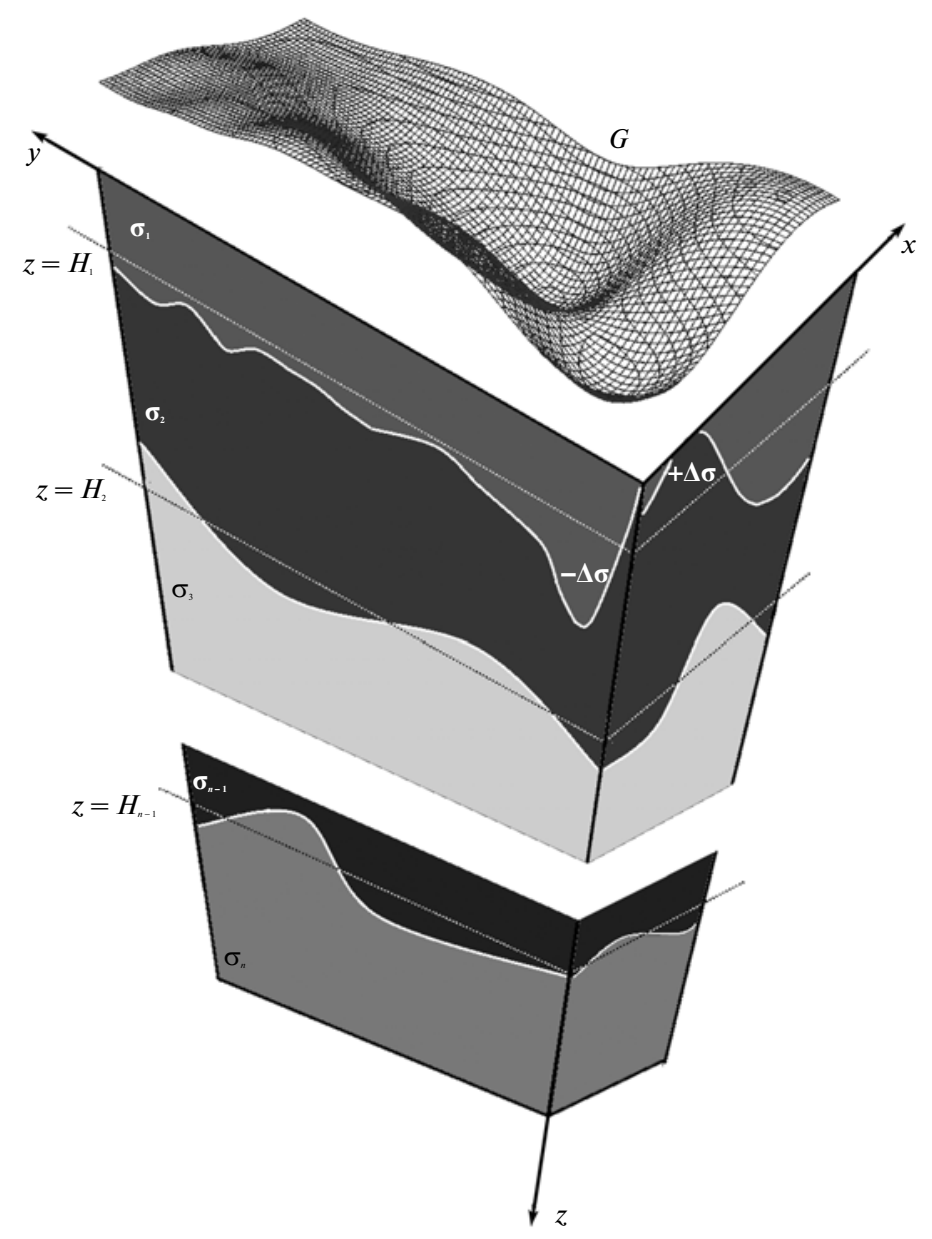

Fig. 1. Model of a multilayer medium.

the linearized steepest descent method (LSDM)

$$
\begin{gathered}
z_{i}^{k+1}=z_{i}^{k}-\gamma_{i} \frac{\left\|S\left(z^{k}\right)\right\|^{2}}{\left\|A^{\prime}\left(z^{k}\right) S\left(z^{k}\right)\right\|^{2}} S_{i}\left(z^{k}\right) \\
S\left(z^{k}\right)=A^{\prime}\left(z^{k}\right)^{T}\left(A\left(z^{k}\right)-F\right)
\end{gathered}
$$

and the linearized minimum error method (LMEM)

$$
z_{i}^{k+1}=z_{i}^{k}-\gamma_{i} \frac{\left\|A\left(z^{k}\right)-F\right\|^{2}}{\left\|S\left(z^{k}\right)\right\|^{2}} S_{i}\left(z^{k}\right),
$$

where $\gamma_{i} \in[0,1], z_{i}$ is the $i$ th component of resultant vector $z(x, y)$, and $k$ is the iteration number.

Weight factors $\gamma_{i}$ will be chosen as follows:

$$
\begin{aligned}
F=\left[F_{1}, F_{2}, \ldots, F_{L}\right] & =\left(f_{1}, f_{2}, \ldots, f_{M \times N}, \ldots, f_{L \times M \times N}\right) \\
\rightarrow & \left(\gamma_{1}, \gamma_{2}, \ldots, \gamma_{L \times M \times N}\right), \\
\gamma_{i} & =\frac{\left|f_{i}\right|^{\beta}}{\max _{i}\left|f_{i}\right|^{\beta}}, \quad \beta>1,
\end{aligned}
$$

where $F_{l}(l=1,2, \ldots, L)$ are anomalous fields generated by the gravitating mass located below the corre- sponding depths $H_{l}$ for the sought surfaces of interface $S_{l}(l=1,2, \ldots, L)$.

Weight factors $\gamma_{i}$ were found successively from field $F_{L}$ separated from field $F$ using the technique of [2], from field $F_{L-1}$ separated from the difference field $F-$ $F_{L}$, from field $F_{L-2}$ separated from the difference field $F-F_{L}-F_{L-1}$, and so on.

For numerical implementation of methods (2) and (3), the horizontal asymptotic planes $z_{l}^{0}=H_{l}(l=1,2$, ..., $L)$ were used as the initial approximation. The condition $\frac{\|A(z)-F\|}{\|F\|}<\varepsilon$ at sufficiently small $\varepsilon$, where $F=$ $\sum_{l=1}^{L} F_{l}$, was taken as the termination criterion.

The use of multiprocessor computing systems essentially reduces the execution time. The idea of parallelizing algorithms (2) and (3) implies dividing the matrix $A^{\prime}\left(z^{k}\right)^{T}$ into blocks and the solution vector $z(x, y)$ and right-hand side vector $F(x, y)$ into parts. For the current iteration, each of the processors (cores) computes a definite part of the solution vector. 

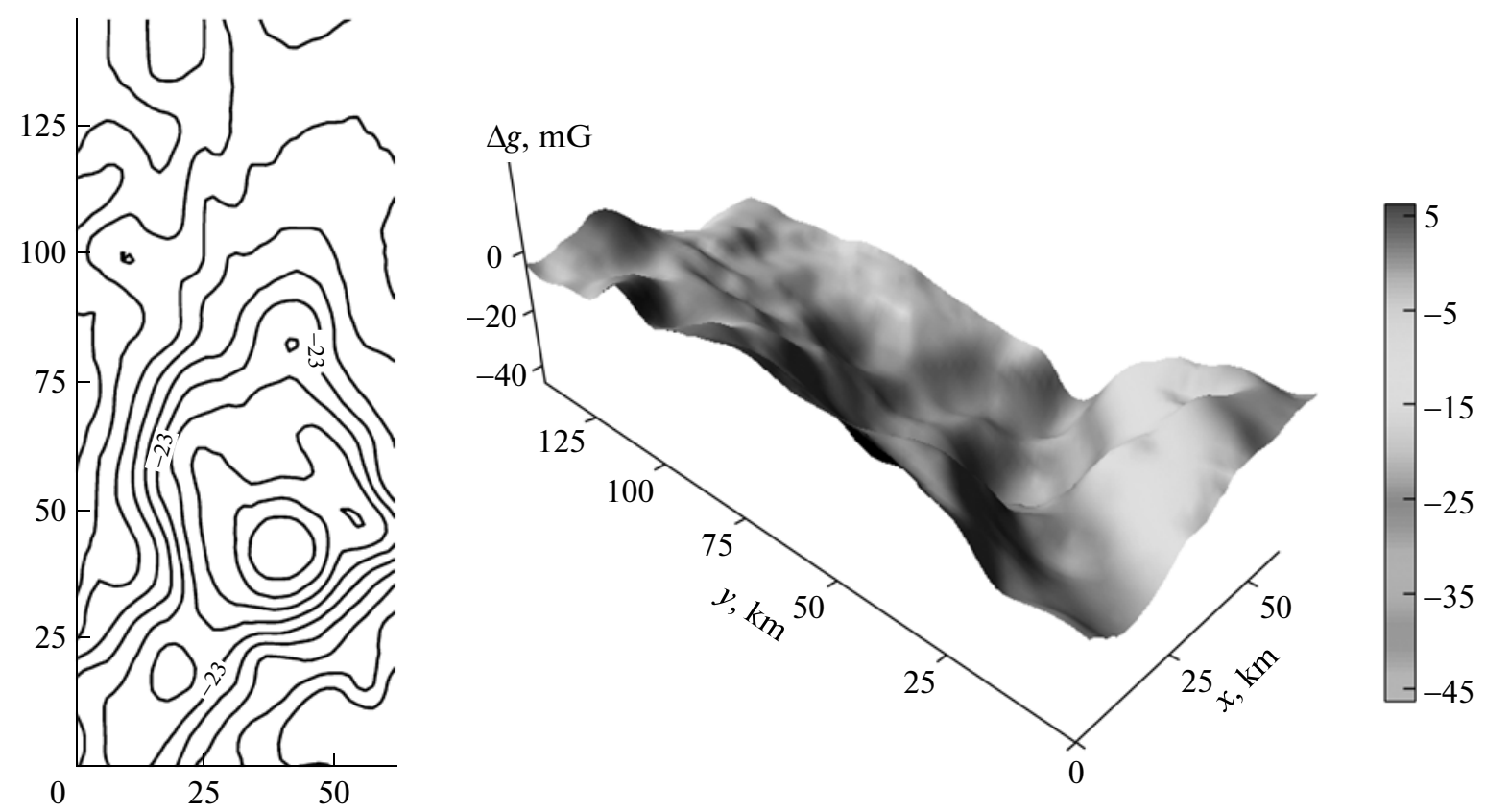

Fig. 2. Observed gravitational field.
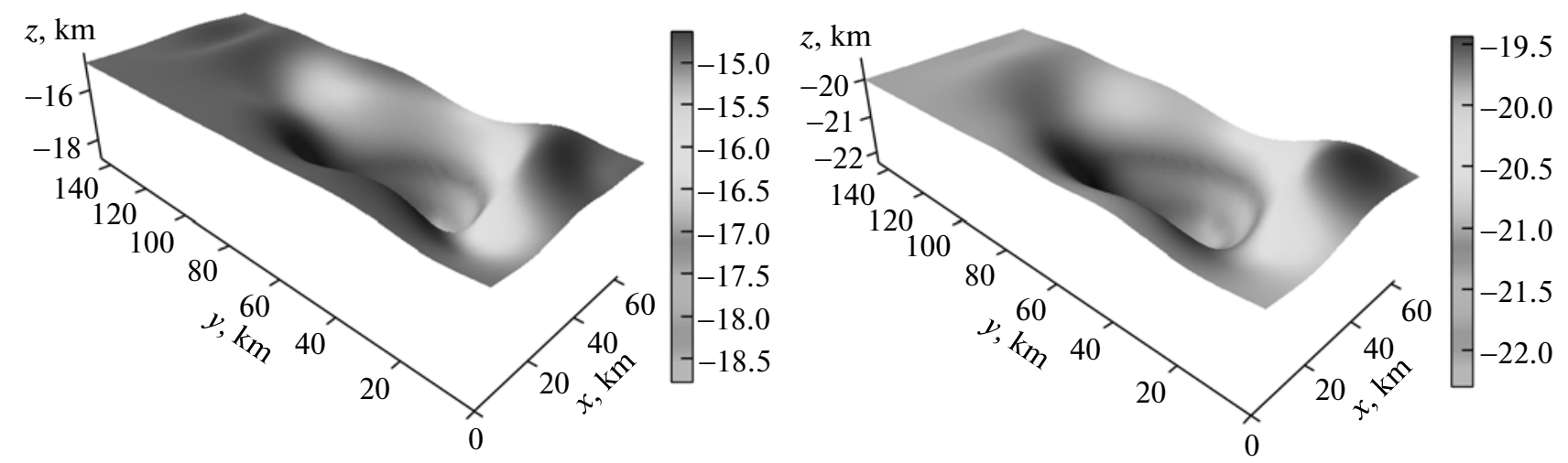

Fig. 3. Interfaces $S_{1}, S_{2}$, at depths $H=5$ and $20 \mathrm{~km}$.

For the eastern Middle Urals, the gravity problem of finding interfaces of $S_{1}, S_{2}, S_{3}$ media with density jumps $\Delta \sigma_{1}=0.3, \Delta \sigma_{2}=0.2$, and $\Delta \sigma_{3}=0.1 \mathrm{~g} / \mathrm{cm}^{3}$ was solved for the model of a four-layer medium using data of [6] measured over an area $S=62 \times 145.75 \mathrm{~km}^{2}$. The distances to the asymptotic planes were taken to be $H_{1}=15, H_{2}=20$, and $H_{3}=25 \mathrm{~km}$. Discretization of Eq. (1) on a $82 \times 108$ grid with steps $\Delta x=0.756, \Delta y=$ $1.35 \mathrm{~km}$ yields vector $z(x, y)$ of dimension 35424 and matrix $A^{\prime}\left(z^{k}\right)^{T}$ of dimension $35424 \times 8856$.

The problem was solved on an Uran supercomputer using the LSDM and LMEM algorithms. As a result, the relative norm of discrepancy $\varepsilon$ decreased by two orders of magnitude as compared to the initial $\varepsilon_{0}=1$. As compared to the solution of problems for fields distinguished for each structural boundary using methods (3) and (4) with factor $\gamma=0.05$ for $\varepsilon=0.015$ (number of iterations $N=288$ ), the solution of gravity problems for the sum of fields by methods (3) and (4) with weight factors $\gamma_{i}$ at $\beta=1.4$ for $\varepsilon=0.015$ was reached with fewer iterations $N=70$. Using model examples, it was demonstrated that solution of problem (1) for the sum field provides an order of magnitude decrease in the relative error, number of iterations, and execution time as compared to that for each structural boundary.

The time of solving the gravity problem by the LMEM method on six-core Intel processors or on an NVIDIA Tesla GPU was 73 and 36 s, respectively.

Figure 2 demonstrates the observed gravitational field. Figures 3 and 4 show the interfaces found. 


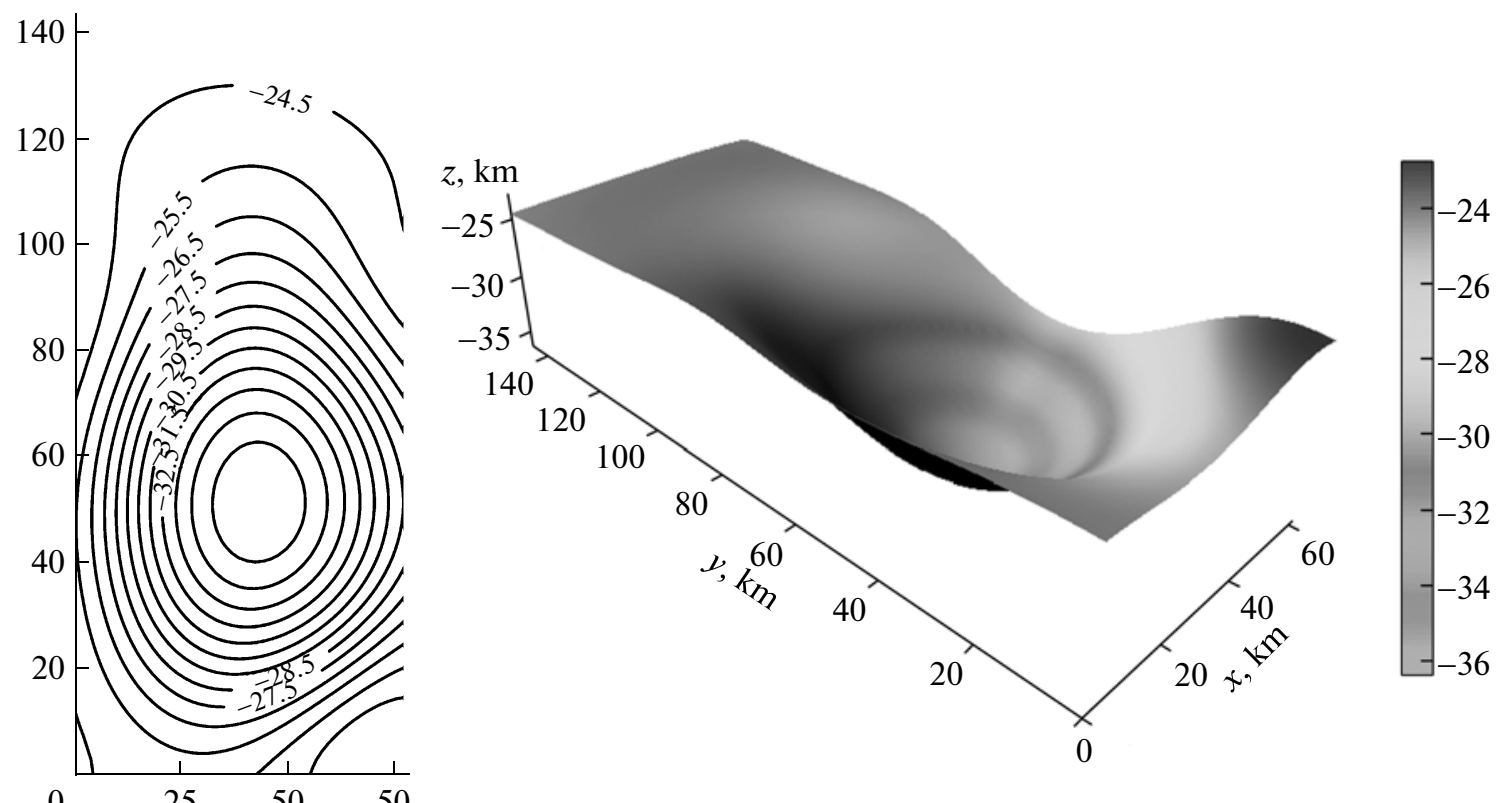

Fig. 4. Interface $S_{2}$ at the depth $H=25 \mathrm{~km}$.

Note that the geomagnetic data for the same area $\mathrm{S}$ were interpreted by collaborators of the Institute of Geophysics, Ural Branch, Russian Academy of Sciences, and taken into account for choosing the medium model considered above.

\section{ACKNOWLEDGMENTS}

We are grateful to V.A. P'yankov for kindly providing data and discussion of the results.

This work was supported by the Ural Branch of the Russian Academy of Sciences within the framework of the Program of Fundamental Research of the Presidium of the Russian Academy of Sciences (program no. 18 , project 12-P-15-2019).

\section{REFERENCES}

1. P. S. Martyshko, I. V. Ladovskii, and A. G. Tsidaev, Izv., Phys. Solid Earth 46 (11), 931-943 (2010).

2. P. S. Martyshko and I. L. Prutkin, Geofiz. Zh. 25 (3), 159-168 (2003).

3. V. V. Vasin, G. Ya. Perestoronina, I. L. Prutkin, and L. Yu. Timerkhanova, Mat. Model. 15 (2), 69-76 (2003).

4. E. N. Akimova, Vestn. NNGU, No. 4, 181-189 (2009).

5. V. V. Vasin and G. G. Skorik, J. Inverse Ill-Posed Problems 18 (8), 855-876 (2011).

6. P. S. Martyshko, V. V. Vasin, E. N. Akimova, A. L. Rublev, V. A. Pyankov, in Proceedings of the 11th Conference on Geoinformatics: Theoretical and Applied Aspects, Kiev, Russia, 2012 (Kiev, 2012).

Translated by M. Bogina 